\title{
Tangence
}

\section{Statut de l'intime et du vrai dans une littérature en émergence : le cas des Mémoires de Pierre de Sales Laterrière (1743-1815)}

\section{Bernard Andrès}

Numéro 45, octobre 1994

Authenticité et littérature personnelle

URI : https://id.erudit.org/iderudit/025828ar

DOI : https://doi.org/10.7202/025828ar

Aller au sommaire du numéro

Éditeur(s)

Tangence

ISSN

0226-9554 (imprimé)

1710-0305 (numérique)

Découvrir la revue

Citer cet article

Andrès, B. (1994). Statut de l'intime et du vrai dans une littérature en émergence : le cas des Mémoires de Pierre de Sales Laterrière (1743-1815).

Tangence, (45), 91-106. https://doi.org/10.7202/025828ar 


\title{
Statut de l'intime et du vrai dans une litté- rature en émergence : le cas des Mémoires de Pierre de Sales Laterrière (1743-1815) ${ }^{1}$
}

\author{
Bernard Andrès
}

Pour le chercheur désireux de constituer un corpus littéraire dans une période aussi problématique que celle de la fin du $x_{1 I I}{ }^{\mathrm{e}}$ siècle québécois, l'écrit intime offre des ressources insoupçonnées. Qu'il s'agisse de correspondances privées, de documents notariés, de minutes de réunions, de notes ou de relations de voyages, de journaux intimes, de poésies de circonstance ou de mémoires destinés à la famille du scripteur, ces textes figurent à juste titre dans le catalogue des premiers écrits, de la Conquête au tournant du XIX ${ }^{e}$ siècle. Ils n'ont pas, pour la plupart, accédé à la sphère publique, ni fait l'objet d'aucune diffusion dans les presses naissantes de la "Province of Quebec", puis du "BasCanada". Mais leur existence dans les fonds d'archives atteste déjà chez leurs auteurs d'une certaine familiarité avec la chose écrite et, parfois, d'une maitrise certaine des discours à la mode, ou des écrits des "grands" de ce monde ${ }^{2}$. Ces écrits intimes aujourd'hui redécouverts provoquent aussi un effet d'authenticité qu'il convient toutefois d'interroger lorsqu'il s'agit de témoignages ponctuels ou de mémoires sur l'époque. Quel crédit accorder aux souvenirs de l'officier, du notaire, du vieux médecin ou du maitre d'école qui relate son passé sur fond d'événements historiques? Comment s'ébauche la chronique d'une vie, se bâtit aux confins du vécu et du rêvé, du réel et de la fiction, le "récit de soi "3?

1 Ce travail est mené dans le cadre du projet "Archéologie du littéraire au Québec. (ALAQ), subventionné par le CRSHC. Une version antérieure de ce texte a été présentée à l'Université du Québec à Trois-Rivières, au Regroupement des chercheurs sur l'usage des écriture intimes du littéraire et du social (RECUEILS), le 23 septembre 1993.

2 Nous avons rassemblé et présenté un certain nombre de ces textes dans $\mathrm{La}$ conquête des Lettres au Québec (1764-1815): Florilège (en collaboration avec Pascal Riendeau), Montréal, UQAM, Département d'études littéraires, Cahiers de l'ALAQ, $\mathrm{n}^{\circ} 1$, mars 1993, $446 \mathrm{p}$.

3 Pour reprendre l'expression de Simon Harel dans le récent numéro de Tangence, $\mathrm{n}^{\circ} 42$, décembre 1993. 
92

La valeur accordée de nos jours à ces témoignages dépend d'abord de leur statut historiographique ou littéraire. Leur "vérité " repose sur le sort que l'institution leur réserve dans le panthéon des textes. Annales ou fictions? Dans un cas, ils sont retenus comme simples traces du passé, servant à l'historien de base documentaire dans une étude comparative des pratiques sociales ou des mentalités. Aucun d'entre eux n'est plus véridique que l'autre; c'est du croisement des annales que nait la "vérité " historique, au terme d'une opération démonstrative et d'une réénonciation des annalistes par l'analyste. Dans l'autre cas, ils sont tenus pour des fictions "littéraires" (avec la péjoration du terme également rencontrée dans l'épithète "romancé " ${ }^{4}$ ). Aussi convient-il, avant d'aborder l'un d'entre eux sous l'angle de sa "véracité", de préciser le corpus dont il relève.

Les documents épars auxquels nous nous intéressons s'avèrent surtout connus de l'archiviste et de l'historien. Ils n'ont pas encore accédé à la reconnaissance des littéraires. Pour les amateurs de "Belles Lettres", pour les auteurs de manuels, ils n'ont, au mieux, qu'un statut auxiliaire (ils aident à comprendre d'autres écrits "majeurs"). Une valeur accessoire leur est parfois octroyée dans la hiérarchie des productions d'un auteur: avant l'CEuvre, ou après, ou à côté d'Elle, ce sont les brouillons, avanttextes, correspondances et autres écrits mineurs aux mérites jugés bien relatifs, surtout lorsqu'ils relèvent de l'„intime". Est-il possible de concéder un autre statut à ces OVNIS de l'écriture ("Euvres virtuelles non identifiées")? C'est ce que je tenterai bientôt à propos du statut de l'intime et du vrai dans les Mémoires de Laterrière. C'est plus largement le pari que nous tenons à l'ALAQ en sortant de l'oubli un certain nombre de vestiges sripturaires dont l'aspect documentaire nous intéresse moins que la valeur discursive intrinsèque ${ }^{5}$. Il ne s'agit pas à proprement parler d'une valeur "littéraire" jugée à l'aune des "chefs-d'œuvre", mais de la capacité de ces textes de s'inscrire dans des formations discursives précises: le discours des Lumières, de la Révolution,

4 "Mémoires romancés", dira, non sans condescendance, Ægidius Fauteux à propos du texte de Pierre de Sales Laterrière, comme on le verra.

5 Nous entendons, dans l'optique foucaldienne, transformer ces documents en monuments; $c f$. Bernard Andrès, "De l'archive au corpus: problématique d'une archéologie du littéraire au Québec: (1764-1815)", dans Principes du littéraire au Québec (1766-1815), sous la direction de Bernard Andrès, Cahiers de l'ALAQ, no 2, août 1993, p. 11-12. 
de l'identité ou de l'identitaire, soit en l'incorporant, ce discours, soit en s'en démarquant. On pense d'une part aux écrits de Jautard, du Calvet, Mézière, Bailly de Messein ou Laterrière: autant de variations sur le thème de la liberté de pensée, autant de réflexions sur la quête d'une identité, au plan personnel, ou d'un identitaire, au plan collectif. On pense aussi, a contrario, aux écrits de $M^{g r}$ Briand, de Simon Sanguinet, François Baby ou de Jean-Baptiste Badeaux, au moment de l'invasion américaine. Mais ce qui compte pour nous, ce n'est pas de classer ces écrits en fonction d'un clivage idéologique trop commode (conservateurs ou loyalistes, versus libéraux, ou révolutionnaires). C'est plutôt d'examiner comment ces écritures "naviguent" dans la texture idéologique de l'époque, participent d'une agonistique générale, souvent à l'intérieur d'un même corpus, au sein d'un même regroupement d'intérêts, d'une même famille politique: Quesnel fustige les révolutionnaires et défend la liberté d'expression artistique, Sanguinet dénonce les Canadiens pro-rebelles et se prononce pour une université non confessionnelle. Bailly de Messein, pour sa part, se soumet à l'autorité ecclésiastique, tout en témoignant de son amertume et de ses tourments pendant sa mission en Acadie ${ }^{6}$; plus tard, cet ancien élève du Collège Louis-LeGrand et précepteur des enfants de Carleton n'hésitera pas à s'opposer à son évêque dans une prose digne des Philosophes. Par ailleurs, dans la franco-maçonnerie de l'époque, des tensions existent bien, comme l'a montré Roger Le Moine ${ }^{7}$.

Toutes ces tensions, ces ruptures discursives - au sens propre, ces polémiques - transitent par et s'éclairent dans ces premiers écrits, ces premiers auteurs intimement persuadés d'être dans "le vrai". Même lorsqu'elles relèvent d'un espace privé, ces pages n'en émargent pas moins d'un "discours social" (Angenot) qu'elles travaillent aussi en retour. Mais elles le font sur un mode bien à elles. J'ai l'intuition que ce travail du social dans le texte et du texte sur le hors-texte apparaît avec plus d'évidence dans l'écrit intime que dans la publication. Dans le secret de mes mots, des mots que je me destine ou que j'adresse à mes proches, ne suis-je pas plus libre d'en user à ma guise avec les canons du Discours,

6 Cf. Gilles Lenghan, "Les écrits de Bailly de Messein", communication au Séminaire de l'ALAQ, 15 avril 1993.

7 Roger Le Moine, "Francs-maçons du régime français et de la "Province of Quebec", dans Principes du littéraire [...J, op. cit., p. 30-31. 
94

de la Doxa et de la thétorique? Pourquoi forcer la note, pour qui, dans le silence de l'intime? Alors que publier, c'est toujours, peu ou prou, adopter une posture, se mettre en peine de convertir, séduire, poser pour la postérité, faire œuvre de fiction...

Qu'on m'entende bien: je ne veux pas tomber dans le mythe de la spontanéité. Qu'il s'agisse de corpus québécois ou français, l'authenticité ne réside pas plus dans la voix intime que sur la voie publique. On peut aussi se conter des sornettes dans le privé. Mais on le vit alors sur le mode du fantasme, d'un scénario qui n'engage que soi-même (ou le petit cercle de ses proches). Personne n'est dupe: ni l'auteur qui s'invente une vie, ni les parents ou amis qui le connaissent déjà, qui la connaissent déjà, sa vie et qui liront, devineront dans le bio-graphein l'écart introduit par l'écrivain ou l'épistolier entre l'existences vécue et l'existence rêvée. Il en va autrement du mémorialiste qui destine explicitement son texte à l'impression, à un public inconnu et ignorant, bien souvent, de l'existence en question; un public plus apte par là-même à se laisser leurrer par les rêves de l'autobiographe. Pour ce lecteur lointain, le grand homme prend une posture: nous le voyons tailler sa plus belle plume, aiguiser son ciseau, jauger le marbre dont il fera sa propre statue. Et même s'il condescend à évoquer ses faiblesses passées, à conter l'épisode honteux du ruban volé (Rousseau), ou ses pires "jours de tribulations" (Chateaubriand), c'est pour mieux te manger, cher lecteur, chère lectrice, mieux te séduire en t'entraînant sur sa pente narrative. Vincent Jouve a bien montré la stratégie du "personnage infériorisé" et "le regard affectueux, presque paternel " qu'elle suscite chez le lecteur ${ }^{8}$. Je crois ce que je crois dominer: ce qu'on me donne à (et que je veux bien) croire. Le dire-vrai de l'auteur repose sur un faire paraitre vrai dont Greimas et Courtés ont bien montré le dispositif véridictoire: "un croire-vrai doit être installé aux deux extrémités du canal de la communication, et c'est cet équilibre, plus ou moins stable, cette entente tacite de deux complices plus ou moins conscients que nous dénommons contrat de véridiction" "?

L'analyse des effets de sens "vérité" dans les discours qui nous intéressent repose en fait sur leur contexte énonciatif, d'où la nécessité de retracer au préalable ce que j’appelle ailleurs la sociogénèse

8 Vincent Jouve, L'effet-personnage dans le roman, Paris, PUF, 1992, p. 180.

9 A. J. Greimas, J. Courtés, Dictionnaire raisonnée de la théorie du langage, Paris, Hachette, 1979, p. 417. 
de leur énonciation ${ }^{10}$. Non plus ce qu'ils nous apprennent ou nous cachent de la vie du mémorialiste et de son époque, mais comment. La question est alors de savoir selon quelles stratégies énonciatives se fonde la vérité de ces récits : le type de rapport qu'ils entretiennent avec le narrataire, avec le discours social ambiant, avec ce qui se dit (peut se dire) dans la société de référence.

Précisons d'emblée que nos textes témoignent d'une relation problématique avec le "réel ", et ce à deux niveaux: générique et historique. Le genre même de l'autobiographie, dont Philippe Lejeune a bien montré les apories ${ }^{11}$, ne saurait reproduire platement une donnée existentielle préexistante. Dans son récent travail sur l'autobiographie, Barbara Havercroft a relevé les “ interférences [qui] viennent troubler la reprise autobiographique ": trous de mémoires, fiction, rêve, invention, discours d'autrui, etc. interdisent à ce type d'écriture de se donner comme "une copie exacte d'une sensation ou d'un événement antérieur "12. Au plan historique, fait tout aussi problème le rapport de nos textes avec le "réel" de l'époque: celui d'un territoire en pleine mutation où "nouveaux" et "anciens" sujets s'interrogent à partir de discours (au départ) diamétralement opposées sur la nation, la patrie et l'Autre. Au sein même des francophones comme des anglophones, s'opposent les tenants de l'assimilation, de l'intégration, du maintien ou du rejet de l'aristocratie, adeptes du statu quo économique ou du grand négoce pan-américain, du respect de l'autorité coloniale, ou de la subversion politique. Qui détient la "vérité " dans les débats sur l'invasion américaine ou l'avenir constitutionnel? Et surtout, dans notre optique, selon quelles stratégies énonciatives? Comment, où et avec qui, en parler? Dans le secret des journaux intimes, le repli des correspondances, ou dans cet "espace public" en formation que le nouveau pouvoir met en place sous l'impulsion de la bourgeoisie marchande?

Avec l'imprimerie et les gazettes, de nouveaux espaces d'écriture voient en effet le jour et, pour la première fois des Canadiens s'expriment publiquement ${ }^{13}$, signent des pétitions, éditent

10 Bernard Andrès, "De l'archive au corpus... ", op. cit., p. 14.

11 Philippe Lejeune, Moi aussi, Paris, Seuil, 1986, p. 34 et s.

12 Barbara Havercroft, "L'autobiographie comme reprise: l'exemple d'Enfance de Sarraute ", Tangence, n ${ }^{\circ}$ 42, décembre 1993, p. 132.

13 Ces prises de position publiques d'individus sur les affaires d'état étaient difficilement imaginables sous le Régime français. Il eśt significatif que Daniel 
96

leurs propres textes. De nouvelles choses peuvent se dire différemment. Mais pour quelques notables enhardis, pour un SaintLuc de La Corne qui publie sous son nom son journal de voyage $^{14}$, combien d'anonymes et de pseudonymes chez les premiers gazetiers, tels "Le Canadien curieux", "Le Sincère", "Le Bon Sens", "Un Citoyen", "L'Homme Mûr", ou d' Athanase Cul-deJatte "? Manière de déjouer la censure, ou façon encore timide d'aborder la sphère publique? Les deux probablement. Avec, pour la fraction instruite des Canadiens, cette possibilité toute nouvelle de reconfigurer les territoires de l'intime et du privé, en fonction d'un espace commun d'échanges discursifs plus vaste et mieux publicisé que sous le régime précédent. Presses naissantes, journaux, lettres ouvertes, pétitions: autant de lieux nouveaux où s'exerce l'écrit, qui sollicitent l'individu et le forcent à s'impliquer en dehors de l'ancienne sphère privée (couple, famille, rang, paroisse). Toute sociale et marquée par l'histoire est la façon dont le " $\mathrm{JE}$ " tente alors de préserver, au cour du privé, son intimité.

En effet, comme le rappelle Manon Brunet, l'intime se définit déjà "comme une activité sociale"15. En ce qu'il témoigne d'une distance prise face à l'environnement et qu'il se distingue d'un "privé" moins fermé à autrui, l'intime relève bien d'un processus d'insertion. Ajoutons: surtout devant la menace d'une insertion forcée. Intime et identitaire s'entendent alors de concert. Au lendemain de la Conquête, face à une possible assimilation vécue comme atteinte à l'intime, le "nouveau sujet" Canadien ne peut préserver son identité qu'en se définissant par rapport à l'uancien": l'Anglais vient en effet, comme bien l'a vu Simon Harel, "structurer ce pays informe par la constitution d'une identité rigide et défensive" ${ }^{16}$. Ainsi, paradoxalement, c'est pour préserver une intimité menacée que le sujet canadien se lance dans l'arène publique, prend position, s'exposant par là-même à y rencontrer

Latouche et Diane Poliquin-Bourassa fassent débuter leur anthologie de ce type de textes en 1760 (cf. Le manuel de la parole. Manifestes québécois, Tome 1. 1760 à 1899, Sillery-Québec, Boréal Express, 1977).

14 Luc Saint-Luc de La Corne, Journal du voyage de $M$. Saint-Luc de La Corne, ecuyer, dans le navire l'Auguste, en l'an 1761, Montréal, Fleury Mesplet, $1778,38 \mathrm{p}$.

15 Manon Brunet, "Le territoire de l'intime", dans Manon Brunet et Serge Gagnon éd., Discours et pratiques de l'intime, Québec, IQRC, p. 10

16 Simon Harel, Le voleur de parcours. Identité et cosmopolitisme dans la littërature québécoise contemporaine, Longueuil, Balzac, p. 95. 
l'Autre... et à s'y découvrir autre, autrement. C'est l'ère nouvelle de l'écrit, du verbe échangé dans le bilinguisme hésitant des gazettes; c'est l'argumentation dans les nouvelles sociétés de débats qui figurent un peu, si l'on y pense bien, des réseaux intimes de discussion publique. On songe, bien sûr aux loges maçonniques où se risquent à présent des francophones et qui, dans le secret des tenues, conjuguent intime et fraternité. Mais audelà de ces circuits plus discrets, c'est aussi toute une vie associative où des individus (qui excèdent l'ancienne élite aristocratique) font désormais cercle et, dans l'intimité de la coterie, fondent autant de "sociétés de convivialité": clubs, académies et cercles littéraires ${ }^{17}$. Pour le Canadien qui s'y mêle, une intégration à la sphère publique s'ébauche alors, dans une intimité nouvelle (et parfois conflictuelle, qu'importe?) avec l'Autre. C'est dans ce nouveau contexte qu'il convient d'apprécier le statut de l'intime et son rapport au vrai dans les textes qui nous préoccupent.

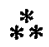

Qu'en est-il, plus précisément des mémoires, genre hybride de récit du moi, où l'auteur, se narrant, raconte aussi bien son époque, l'accent étant mis sur les événements historiques dont il fut le témoin ou l'acteur? Que dire de ce témoignage de soi et des autres où, contrairement au discours historique, "les choses vues ont le pas sur la consultation des sources et des archives" ${ }^{18}$ ? Si la subjectivité assumée du mémorialiste prime sur l'objectivité (présumée) de l'historien, la question du vrai se pose de façon tout à fait singulière dans ce discours a priori non fictif où le JE livre son intimité sur fond d'espace public. De l'histoire vécue connue d'un seul, ou de l'Histoire officielle connue de tous, laquelle cautionne l'autre, laquelle fonde le vrai? Ce que je

17 Cf. Maurice Lemire (dir.), La vie littêraire au Québec. t. 1 (1764-1805). La voix française des nouveaux sujets britanniques (1764-1805), Québec, Presses de l'Université Laval, 1991, p. 161-180 et Pierre Rajotte, "Les pratiques associatives et la constitution du champ de production littéraire au Québec (1760-1867)", Revue d'bistoire de l'Amérique française, vol. $\mathrm{XLV}, \mathrm{n}^{\circ} 4$, printemps 1992, p. 545-572.

18 Sur la complexité de ce genre hybride, aux confins de l'autobiographie et de la chronique historique, voir Georges Gusdorf, Lignes de vie 1. Les écritures du moi, Paris, Odile Jacob, 1991, p. 251. 
98

dis du monde est-il vrai parce que je l'ai vécu moi-même, ou ma vie est-elle authentifiée par la notoriété des faits historiques?

Pour apprécier les procédures de véridiction d'un tel discours, j'examinerai le cas des Mémoires de Pierre de Sales Laterrière et de ses traverses. Publié de façon posthume en 1873, ce texte encore peu connu de l'histoire littéraire québécoise fut rédigé vers 1812 , époque à laquelle s'ébauchait en France un tout autre monument littéraire dont je dirai d'abord quelques mots: les Mémoires d'outre-tombe de René de Chateaubriand. Aux antipodes de l'espace et de l'institution littéraire, au pinacle de celle-ci, ou dans sa lointaine périphérie, deux mémoires posent à leur façon la question du vrai dans l'écriture de soi. Il suffit pour s'en convaincre de relire sous cet angle les roueries de Chateaubriand, maître de l'oximore et de la métaphore filée, dans l'avant-propos des Mémoires d'outre-tombe: "mon berceau a de ma tombe, ma tombe a de mon berceau". En 1846, au moment où il rédige ces lignes, l'auteur a 78 ans; il mourra deux ans plus tard, aigri (mais comment y croire?) par la publication anthume d'une ouvre au titre posthume :

La triste nécessité qui m'a toujours tenu le pied sur la gorge m'a forcé de vendre mes Mémoires. Personne ne peut savoir ce que j'ai souffert d'avoir été obligé d'hypothéquer ma tombe [...]; mon dessein était de les laisser à $M^{\text {me }}$ de Chateaubriand; elle les eût fait connaître à sa volonté, ou les aurait supprimés, ce que je désirerais plus que jamais aujourd'hui.

[...] Enfin, si j'étais encore maître de ces Mémoires, ou je les garderais en manuscrit, ou j'en retarderais la parution de cinquante années. ${ }^{19}$

Malgré toutes ses protestations d'innocence, comment penser que ce géant des Lettres françaises ne voyait pas d'un bon œil la divulgation de son ouvre maîtresse, et qu'il eût vraiment préféré lui préserver jusqu'au bout le statut de l'écrit intime? Chateaubriand met trop bien en scène le pathétique de l'écriture, pour résister à publier de son vivant:

[...] les tempêtes ne m'ont laissé souvent de table pour écrire que l'écueil de mon naufrage.

19 Ces citations comme les suivantes proviennent de René de Chateaubriand, Mémoires d'outre-tombe [1850], Paris, ministère de l'Éducation nationale, 1972 , p. 5-8. 
[...] je préfère parler du fond de mon cercueil; ma narration sera alors accompagnée de ces voix qui ont quelque chose de sacré, parce qu'elles sortent du sépulcre.

[...] la vie me sied mal; la mort m'ira peut-être mieux.

Le prurit de publication de Chateaubriand est trahi par l'excès même de la métaphore macabre: "[...] je désirerais ressusciter à l'heure des fantômes, pour corriger au moins les épreuves". Et il achève par une parodie d'écrit intime, celle des dernières volontés consignées dans le plus privé des actes d'écriture: le testament. Parodie, car ce passage d'un goût douteux cèle en fait l'avant-propos d'une ouvre dont il avait déjà publié des extraits en 1834, dont il avait vendu les droits en 1836 , dont il touchait depuis en échange une confortable pension viagère, ouvre enfin que les propriétaires avaient commencé à publier en feuilleton dans La Presse, jugeant que l'homme tardait décidément à mourir. Voici donc cette parodie d'écrit intime livrée en pâture au grand public:

[...] Si je décède hors de France, je souhaite que mon corps ne soit rapporté dans ma patrie qu'après cinquante ans révolus d'une première inhumation [...]. Un cadavre courant la poste me fait horreur; des os blanchis et légers se transportent plus facilement: ils seront moins fatigués dans ce dernier voyage que quand je les traînais çà et là chargés de mes ennuis.

À côté de ces roueries littéraires, ce qu'on a pu qualifier d'excès et de "gasconnades" dans les Mémoires de Laterrière, fait figure d'innocente bluette. J'ai retracé ailleurs la réception critique de cet ouvrage par Benjamin Sulte, Egidius Fauteux et quelques autres détracteurs de Laterrière ${ }^{20}$. Ainsi de Malchelosse en 1960:

Lorsque Laterrière écrivit ses Mémoires [...], il avait dû certainement lire un livre étrange intitulé Appel à la justice, par Pierre du Calvet. Nos écrivains ont tiré de ces deux ouvrages aussi mensongers que possibles des renseignements faux qui s'étendent sur la période de 1764 à 1784 . Laterrière, Français comme Du Calvet et partisan comme lui de la cause américaine, a trop cru celui-ci. Mais Du Calvet, Cazeau, Roubeau, étaient une trinité de fourbes. ${ }^{21}$

20 Bernard Andrès, "La réception de 1" étrange" au Québec. Pierre de Sales Laterrière (1743-1815) ", Critique et littérature québécoise, Annette Hayward et Agnès Whitfield éditrices, Montréal, Triptyque, 1992, p. 199-216

21 Gérard Malchelosse, "Mémoires romancés ", Les cabiers des dix, $\mathrm{n}^{\circ} 25,1960$, p. 134 ; même référence pour la citation de Fauteux (p. 120). 
100

Pourtant, comme nous le verrons, Laterrière n'entendait point au départ faire ouvre de vérité en s'adressant à ses proches. Quant au recours au pathos de la mort, il n'en abuse guère au terme de sa carrière. La seule petite phrase, au dernier chapitre, renvoyant à l'âge de l'auteur est: "J'étais maintenant vieux et infirme". Comment aborder ce texte infiniment plus sobre, en effet, que celui de Chateaubriand, dont le début de la rédaction se situe à la même époque romantique ${ }^{22}$. Mon objet n'étant pas de comparer ces deux " ouvres", mais d'introduire au corpus de nos écrits intimes, je ne formulerai que quelques réflexions sur les Mémoires de Pierre de Sales Laterrière et de ses traverses, rédigés vers 1812-1813, peu avant la mort de l'auteur (1815) et publiés de façon posthume en 1873, dans une "édition intime" d'une centaine d'exemplaires ou moins ${ }^{23}$.

J'ai signalé ailleurs le problème que soulevait ce texte selon qu'on l'appréhende comme mémoires au sens strict (et alors le critère pour la lecture reste la conformité au réel d'une existence inscrite dans l'Histoire), ou comme une fiction des mieux ourdies (Laterrière devenant alors romancier, le critère passant du vrai au vraisemblable de l'histoire racontée). À moins que les mémoires ne puissent échapper à la fiction et que le critère de vraisemblance ne soit lui-même relatif pour ce qui est du récit de vie, lorsqu'il est destiné au premier chef à des proches ${ }^{24}$. C'est ce qui ressort de l'incipit de ces mémoires.

Quoique ma vie n'ait été qu'un tissu continuel de traverses comiques et tragiques depuis que j'ai quitté la maison paternelle, je n'aurois jamais voulu en occuper le public, faute de

22 Chateaubriand aurait entrepris ses mémoires en 1811 (d'après la date figurant sur l'édition de 1850). D'autres le situent en 1809 ou 1803.

23 Sur l'histoire de ces mémoires, René Beaudoin, "Pierre de Sales Laterrière, médecin, mémorialiste et prototype de l'aventurier des lettres ", Principes du littéraire [...], op. cit., 1993, p. 47-49. Pour ce qui est de l'établissement du texte, disons, pour faire bref, qu'en l'absence du manuscrit original (version I), je me fonde pour les variantes, par rapport à l'édition 1873 (version IV), sur la transcription manuscrite effectuée par le petit-fils de Laterrière entre 1855 et 1857 (version II). Les renvois aux Mémoires se feront ainsi : II, 1 (page 1 de la transcription de 1855-1857); IV, 5 (page 5 de l'édition de 1973), etc. Rappelons enfin que Danny Tremblay, de l'ALAQ, a entrepris de comparer les versions II et IV du premier chapitres des Mémoires.

$24 C f$. Bernard Andrès, "De la Conquête au xix ${ }^{e}$ siècle: le cas de Pierre de Sales Laterrière "Écrire le Québec: de la contrainte à la contrariété, Montréal, XYZ éditeur, 1990, p. 61-77. 
talens suffisants et n'étant point écrivain ni historien; mais mes chers enfans me demandent que je leur laisse des mémoires qui les instruisent de... 25

Je ne puis croire, en effet, que les "chers enfans" auxquels le patriarche Laterrière destinait ses "notes" n'eussent pas déjà une petite idée de la vie et des rêves de leur géniteur quand ils lui demandèrent de rédiger ses souvenirs ${ }^{26}$. Pour eux, le problème de la vérité ne se pose pas, ou alors de façon toute relative, compte tenu de la connaissance directe qu'ils ont déjà du biographé en question. C'est bien plus tard, pour les lecteurs des années 1870 influencés par Benjamin Sulte, que le dire-vrai de Laterrière ne sera plus tout à fait cru-vrai. Car, en 1812, le vieux médecin ne se doute pas que, soixante ans plus tard, nul autre que l'abbé Casgrain transformera ce "ramassis" (expression de Laterrière) en manuscrit, puis en livre. Fait significatif, devenue notable en 1812, le nouveau seigneur des Éboulements, n'est pas convaincu, d'entrée de jeu, de ses capacités d'écriture. À propos du "tissu continuel de traverses tragiques et comiques" de sa vie (IV, 5), il affirme qu'il n'aurait "jamais voulu en occuper le public, faute de talens suffisants et n'étant point écrivain ni historien ". Coquetterie d'écrivain? Peu probable, si l'on en juge par les variantes de la version II : elles révèlent effectivement un nombre important de maladresses stylistiques qui disparaîtront de la version finale révisée par Alfred Garneau (et probablement supervisée par Casgrain). Pourtant, malgré ce manque d'assurance, Laterrière va de l'avant et "divis[e] par chapitres le corps de [s]es notes, afin que, si quelqu'un des mes fils veut les commenter en vue de les livrer à l'impression, il puisse les disposer plus aisément dans un meilleur ordre" (IV,5). La faible probabilité que cela se fasse un jour n'empêche pas le scripteur de fantasmer déjà un narrataire extra-familial: "Mes chers enfans m'excusent d'avance; il ne me reste plus qu'à prier le lecteur étranger de vouloir porter la générosité jusque-là que d'en faire autant " 27 . Je n'ai guère le loisir de

25 Mémoires, IV, 5 (les points de suspension renvoient à la note suivante d'Alfred Garneau: "Le papier déchiré ne permet pas de voir la suite ".

26 Les journaux du fils Laterrière (Pierre-Jean) nous éclaireront probablement sur les attentes de la famille à ce chapitre. Voir la publication du premier d'entre eux (1815), que nous venons de donner avec Pierre Lespérance: Fortune et infortunes d'un dandy canadien. Pierre-Jean de Sales Laterrière: Joumal de voyage (1815), Cahiers de l'ALAQ, numéro 3, hiver 1994, $92 \mathrm{p}$.

27 IV,6; noter que la version II, plus proche de l'original, m'apparait ici plus élégante dans ses archaïsmes et son économie syntaxique que celle, plus 
102

suivre le statut de ce narrataire tout au long des mémoires, mais une rapide recension permet de constater que, par la suite, Laterrière ne mentionne plus que rarement ses enfants comme lecteurs possibles de son texte. Ainsi de ce passage où, après avoir narré l'épisode honteux d'une bonne cuite contractée à Trois-Rivières, le narrateur conclut: "Exemple encore à mes enfans qui liront ce récit des folies de jeunesse de leur père!.. (IV, 92).

En fait, graduellement, c'est la figure d'un "lecteur étranger" qui devient familière au scripteur; on passe ainsi de l'écrit intime à la publication rêvée, du scripteur à l'auteur. Ce narrataire, il le convoque au deuxième chapitre pour amorcer une anecdote sur la mort du docteur Rochambeau, son patron à Paris, vers 1765: "Une remarque, lecteur, qui ne m'est jamais sortie de la mémoire [...]" (IV, 37); le mot "lecteur" apparaît aussi dans (II, 75). Et, deux pages plus loin: "La scène [...] mérite que j'en fasse part au lecteur" (IV,39). Au chapitre suivant, le narrataire est directement interpellé, tutoyé, même: "Lecteur, puisque je te parle ici des indigènes du Canada, il ne faut pas que j'oublie ici de te dire [...]" (IV, 54). Puis, c'est la corrélation explicite entre le "lecteur" et l'" ouvrage". Laterrière s'assume comme auteur: "Comme le lecteur le verra partout dans cet ouvrage [...]” (IV, 93).

L'espace me manque pour analyser plus finement ces glissements progressifs du plaisir d'écrire, de séduire le lecteur et d'ainsi le convaincre d'un dire-vrai autobiographique. C'est l'ascension de l'intime au public et de la séduction à la conviction. Pour la suivre, il faudrait aussi relever le nombre considérable de mentions, dans le récit, de correspondances privées, d'actes notariés et de journaux personnels que le héros dit avoir rédigés, perdus et cités de mémoire dans ses Mémoires. Autant de références dans l'intime à du plus intime, dans le vrai mémorial à l'authentique d'écrits (toujours) plus personnels. Ainsi, à propos de l'épisode de Harvard, où il dut à quarante-six ans refaire sa médecine: "Mon journal, bien circonstancié, fut oublié par moi, à mon départ de Boston, dans une petite cassette, chez le $D^{r}$ Warren, notre professeur d'anatomie. Je vais y suppléer autant que ma mémoire me le permettra après un aussi long espace de

ampoulée, de Garneau: "Mes chers enfans m'excusent d'avance et ne me reste que de prier le lecteur étranger de vouloir porter la générosité d'en faire autant $n$. 
tems" (IV, 150) ${ }^{28}$. Visiblement affecté par cette perte, il rajoute, deux pages plus loin, à propos de la même relation de voyage: "[...] elle vaut la peine que mes enfants la réclament pour la joindre au présent récit et rectifier les inexactitudes qui pourront se glisser sous ma plume" (IV, 152). Ailleurs, ce sont les journaux qu'il regrette de ne pas avoir tenu jadis. À propos de son départ de Saint-Salvy vers l'inconnu, vers l'aventure qui le mènera au Canada: "Si j'avais journalisé dans le temps, je rappellerais quelques particularités des différentes villes que nous rencontrâmes en grand nombre [...]" (IV, 10).

Fait troublant s'il en est, malgré la perte du fameux journal de Boston, le narrateur évoque avec force détails des souvenirs vieux d'un quart de siècle, comme ses cours de physique expérimentale avec le $D^{r}$ Webster à Cambridge (IV, 169 sq). Les dialogues quasi socratiques entre le maître et l'élève sont rapportés au style direct. Il n'est pas jusqu'à la mention "Troisième lecture "(pour troisième leçon) qui n'apparaisse dans les Mémoires (VI, 173)... comme si, à ce point du récit, Laterrière ne faisait que retranscrire ses propres notes de cours! Dans ces pages autobiographiques, l'effet de véracité du texte est à son meilleur.

Ce qui est sûr, c'est que, parvenu à la fin de son existence (et de la rédaction des Mémoires), Laterrière dispose probablement de plus en plus de matériaux intimes (non perdus, ceux-là) ${ }^{29}$. Tout se passe comme si le registre de l'intime, par lequel s'ouvraient les Mémoires, réapparaissait en force à la clôture du manuscrit. On imagine alors une structure enchâssante (celle de l'intime) prenant en étau l'ensemble du récit de vie publié par Garneau-Casgrain. Chassez l'intime, il revient au galop. Sans parler de "retour du refoulé " dans la publication ("intime") de 1873, il faut bien constater la part étonnante des écrits privés dans cet ouvrage.

Au dernier chapitre de ses mémoires, pour évoquer son retour d'Angleterre en 1808, Laterrière écrit carrément: "Je mets

28 Lors de récentes recherches à Harvard, je n'ai pu retrouver la précieuse cassette, mais en revanche, j'ai retracé une correspondance inconnue jusqu'alors de Laterrière avec le président de cette université, le révérend Willard.

29 On retrouve un certain nombre de ces documents dans le fonds de la Seigneurie des Éboulements (APC MG8 F131). 
104

ici le journal que j'ai tenu pendant ce voyage [...]" (IV, 248). Suit une transcription fidèle, jusqu'au style "télégraphique", du périple au jour le jour (à l'heure même): "Cette première nuit, petit vent - Le 17 également. Nous gagnons vers l'ouest [...]. Le 25, à 4 heures du matin, le vent étant repassé au N.-E. [...].. À la toute fin des Mémoires, il cite encore une correspondance privée avec le curé Marcheteau qui avait intercédé dans la vente des Éboulements. Le renvoi se fait dans la plus pure tradition universitaire: "[...] huit jours après je reçus de lui la réponse fastueuse du 9 décembre 1809 , folio $1^{\text {er }}$ de ses lettres, où il n'étoit question que de mettre en lui une confiance sans bornes" (IV, 266). Le passage est suivi d'un autre renvoi à des "réflexions" que Laterrière aurait alors faites sur cette correspondance, commentaires, dit-il, "qui suivent cet ouvrage". Malheureusement pour nous (et pour Garneau qui le note en bas de page), cette annexe au récit fait défaut ${ }^{30}$. Or, la narration elle-même se clôt sur des points de suspension, donnant fort l'impression d'un manuscrit inachevé. Comme la mention "FIN DES MÉMOIRES" n'est peut-être pas de la main de l'auteur (comment vérifier, puisque la transcription du petit-fils (version II) ne se rend pas jusqu'à là?), force est d'imaginer une suite, ou une autre fin (toute privée, celle-là)... qui nous laisse désespérément sur notre faim. Ajoutons que l'incomplétude même des Mémoires ajoute à leur effet de véracité, dans la mesure où l'inachèvement se donne ici à lire comme la fin de l'auteur (décédé en 1815): on ne triche pas avec la mort.

$$
* *
$$

Ainsi, d'un terme à l'autre du discours mémorialiste, le jeu de l'authenticité se trouve intimement lié au caractère privé des matériaux fondus dans le creuset de la publication. C'est dire l'importance et le statut privilégié des "avant-textes" dans le texte des Mémoires. Il en va d'un effet de lecture fort captivant: l'impression de vivre intensément la vie de l'auteur, grâce à la présence massive de tous ces documents privés. Ils y occupent une fonction hautement stratégique, narratologiquement parlant. Avec

30 René Beaudoin est d'avis que cette annexe aux Mémoires pourrait être le terrier de la propriété récemment acquise par l'auteur (ensemble des documents légaux relatifs à la seigneurie). 
les manques, les trous, les interrogations qu'il suscite, les compléments d'information dont il nous prive ou nous gratifie, l'intime alimente ici ce que Barthes appelait le "code herméneutique" du texte. Toute la dynamique de la lecture repose en effet sur la mise en place d'une énigme, la formulation et la résolution plus ou moins suspendue d'une série de questions à propos du narré. Que dit le texte autobiographique? Que cèle-t-il aussi bien? Comment s'y prend-il pour nous convaincre de sa véracité, protester de son bien-fondé? C'est pour beaucoup en recourant à l'intime qu'il construit son propre procès de véridiction. Car l'intime présuppose un secret plus ou moins bien gardé dont le discours (dé)voile l'existence: montre et cache le mystère, affirme et dénie l'évidence ${ }^{31}$.

Dire le privé, c'est se priver de dire le fin mot de ce qu'on exhibe: ses origines (nobles ou roturières) et, fameux ou infamants, ses apprentissages, son parcours et son point d'arrivée (ou de départ vers l'au-delà). Lire le privé, c'est apprécier ce petit jeu du JE à (dé)couvert, c'est deviner ce qu'on nous cache ainsi (et aussi) ostensiblement. C'est surtout en vérifier la présence/ absence dans le texte, plutôt que la conformité au "réel" (?). Car, en bonne logique, la question de la vérité ou de la fausseté d'un énoncé ne se règle pas (seulement) eu égard au "réel" conçu comme un "référent" externe au discours. En admettant même que ce "réel" existe de façon univoque et unanimement partagée, la conformité du discours à cette hypothétique référent ne suffit pas à fonder le "vrai " discursif. Encore moins le "vrai" narratif. Sans tomber dans une conception immanentiste du texte, convenons que le "dire-vrai" comme le "croire-vrai" sont affaire de convention, tout comme ce "réel" dont la narration s'épuisera toujours à rendre compte. Nous le rappelle un écrivain contemporain qui, tout en refusant la posture du mémorialiste, a toujours joué avec le "réel" et la séduction du biographique :

[...] les mille et une versions, les mille et un visages d'une histoire sont aussi ou plutôt sont, constituent cette histoire, puisque telle elle est, fut, reste dans la conscience de ceux qui la vécurent, la souffrirent, l'endurèrent, s'en amusèrent $[. .$.$] et$

31 Simon Harel parle, lui, du "fantasme héroïque qui vise à détenir, sinon à sceller le "secret " des origines de manière à ce que le récit devienne l'incarnation - rêvée - d'une plénitude existentielle" (op. cit., p. 6). 
106

maintenant, maintenant que tout est fini, tenter de rapporter ce qui s'est passé, c'est un peu comme si on essayait de recoller les débris dispersés, incomplets, d'un miroir [...]. ${ }^{32}$

32 Claude Simon, Le vent, Paris, Minuit, 1957, p. 10. Sur la façon dont l'œuvre de Simon composait avec le "réel", le récit de vie et la "biographie d'un alter ego ", voir mon essai Profils du personnage cbez Claude Simon, Paris, Minuit, 1992, p. 209-175. 